\title{
A CLASS OF RIEMANNIAN HOMOGENEOUS SPACES
}

\author{
ISAAC CHAVEL
}

In M. Berger's classification [1] of normal Riemannian homogeneous spaces of strictly positive curvature, appear various classes of Riemannian homogeneous metrics which can be put on odd-dimensional spheres - but which do not have constant curvature. In this paper, we investigate one of these classes, viz. $\{S U(n+1) \times R / S U(n) \times R\}_{\alpha} \equiv{ }_{d e f} M_{\alpha}^{n}$ (cf. details below). In particular, we (i) calculate the conjugate locus (in the tangent space) of any point, (ii) calculate the totally geodesic submanifolds of constant curvature, and (iii) consider closed geodesics in $M_{\alpha}^{n}$ and their relationship to a lemma of W. Klingenberg [5, Theorem 1].

$\S 1$ concerns itself with the explicit construction of $M_{\alpha}^{n}$, and $\S 2$ is devoted to (i). The key tool of $\S 2$ is the writing of Jacobi's equations of geodesic deviation in the canonical connection of $G / H$. (This connection is not the Levi-Civita connection, but nevertheless has the same geodesics.) For the necessary background, the reader is referred to [3]. In $\S 3$ we dispose of (ii), in $\S 4$ we calculate the pinching of $M_{\alpha}^{n}$, and in $\S 5$ we discuss (iii). $\S 5$ is a direct generalization of M. Berger's argument in [2, pp. 9-12], and $\S 6$ consists of remarks relating the spaces $\boldsymbol{M}_{\alpha}^{n}$ to problems in Riemannian geometry.

\section{The space $M_{\alpha}^{n}$}

We first let $E_{j k}$ denote the matrix, whose $r$-th row and $s$-th column are given by $\delta_{j r} \delta_{k s}$, i.e., $E_{j k}$ has a 1 in the $j$-th row and $k$-th column and zeros elsewhere, and we set

$$
\begin{aligned}
& A_{j k}=\sqrt{-1}\left(E_{j j}-E_{k k}\right), \\
& B_{j k}=\quad\left(E_{j k}-E_{k j}\right), \\
& C_{j k}=\sqrt{-1}\left(E_{j k}+E_{k j}\right) .
\end{aligned}
$$

Then a basis of $a_{n}=$ Lie algebra of $S U(n+1)$ is given by $\left\{A_{l, l+1}: l=\right.$ $\left.1, \cdots, n ; B_{r j}, C_{r j}: 1 \leq r<j \leq n+1\right\}$. For a bi-invariant metric on $a_{n}$ we choose

$$
\langle X, Y\rangle=-\frac{1}{2} \operatorname{trace} X Y
$$

Received January 23, 1969. Partially supported by NSF Grant GP-5760. 
One then calculates an orthonormal basis of $a_{n-1} \subset a_{n}$ (where $a_{n-1}$ is imbedded in $a_{n}$ in the usual manner) to be $\left\{S_{j}: j=1, \cdots, n-1 ; B_{r j}, C_{r j}: 1 \leq r<j\right.$ $\leq n\}$ where

$$
\begin{aligned}
& S_{j}=\left(1 / \alpha_{j}\right) \sum_{l=1}^{j} l A_{l, l+1}, \\
& \alpha_{j}=\{j(j+1) / 2\}^{1 / 2} .
\end{aligned}
$$

An orthonormal basis of $\mathfrak{m}=$ orthogonal complement of $\mathfrak{a}_{n-1}$ in $\mathfrak{a}_{n}$ is then seen to be $\left\{S_{n}, B_{r, n+1}, C_{r, n+1}: r=1, \cdots, n+1\right\}$. For completeness we list the Lie multiplication table:

$$
\begin{aligned}
& {\left[A_{r j}, A_{k l}\right]=0,} \\
& {\left[A_{r j}, B_{k l}\right]=\delta_{r l} C_{r l}-\delta_{r l} C_{r k}-\delta_{j k} C_{j l}+\delta_{j l} C_{j k},} \\
& {\left[A_{r j}, C_{k l}\right]=-\delta_{r k} B_{r l}-\delta_{r l} B_{r k}+\delta_{j k} B_{j l}+\delta_{j l} B_{j k},} \\
& {\left[B_{r j}, B_{k l}\right]=\delta_{j k} B_{r l}-\delta_{j l} B_{r k}-\delta_{r k} B_{j l}+\delta_{r l} B_{j k},} \\
& {\left[B_{r j}, C_{k l}\right]=\delta_{j l} C_{r k}+\delta_{j k} C_{r l}-\delta_{r l} C_{j k}-\delta_{r k} C_{j l},} \\
& {\left[C_{r j}, C_{k l}\right]=-\delta_{j k} B_{r l}-\delta_{j l} B_{r k}-\delta_{r k} B_{j l}-\delta_{r l} B_{j k} .}
\end{aligned}
$$

As usual, $\left[\mathfrak{a}_{n-1}, \mathfrak{m}\right] \subset \mathfrak{m}$. Furthermore, by direct calculation one obtains

$$
\begin{aligned}
{\left[a_{n-1}, S_{n}\right] } & =0, \\
\operatorname{dim}\left[a_{n-1}, B_{1, n+1}\right] & =2 n-1 .
\end{aligned}
$$

One therefore obtains: $A d(S U(n))$ acts transitively on the unit sphere in $\mathrm{m}$ spanned by $\left\{\boldsymbol{B}_{j, n+1}, C_{j, n+1} ; j=1, \cdots, n\right\}$.

We now consider the direct orthogonal sum of $a_{n} \oplus \boldsymbol{R}=\mathfrak{g}_{n}, \boldsymbol{R}=$ real numbers, with $D$ a basis element of $R$ of length $1,\left[a_{n}, R\right]=0$, and set

$$
\begin{aligned}
\mathfrak{h}_{\alpha} & =\text { linear } \operatorname{span}\left\{S_{1}, \cdots, S_{n-1}, \cos \alpha \cdot S_{n}+\sin \alpha \cdot D, B_{r j}, C_{r j}: 1 \leq r<j \leq n\right\}, \\
\mathfrak{m}_{\alpha} & =\text { linear } \operatorname{span}\left\{\sin \alpha \cdot S_{n}-\cos \alpha \cdot D, B_{j, n+1}, C_{j, n+1}: 1 \leq j \leq n\right\},
\end{aligned}
$$

$0<\alpha \leq \pi / 2, G_{n}=\exp \mathfrak{g}_{n}, H_{\alpha}=\exp \mathfrak{h}_{\alpha}$, and $M_{\alpha}^{n}=G_{n} / H_{\alpha}$, where $\exp$ denotes the exponential map of the Lie algebra to the Lie group it generates. $\pi: G_{n} \rightarrow M_{\alpha}^{n}$ denotes the canonical projection, and $d \pi$, the induced linear map, identifies $\mathfrak{m}_{\alpha}$ with the tangent space of $M_{\alpha}^{n}$ at $o=\pi\left(H_{\alpha}\right)$. A Riemannian metric on $M_{\alpha}^{n}$ is obtained by restricting the metric on $\mathfrak{g}_{n}$ to $\mathfrak{m}_{\alpha} \times \mathfrak{m}_{\alpha}$ and then translating with $G_{n}$. Of course $M_{\alpha}^{n}$ is now Riemannian homogeneous; also $M_{\alpha}^{n}$ is topologically a sphere. From $(1,2)$ we have

Proposition 1. $A d\left(H_{\alpha}\right)$ is transitive on the unit tangent sphere of the orthogonal complement of $\sin \alpha \cdot S_{n}-\cos \alpha \cdot D$ in $\mathrm{m}_{\alpha}$. 


\section{The conjugate locus of $M_{\alpha}^{n}$}

Of course, by the homogeneity of $M_{\alpha}^{n}$, it suffices to consider the conjugate locus of $o=\pi\left(H_{\alpha}\right)$. An immediate consequence of Proposition 1 is

Proposition 2. The conjugate locus of $o=\pi\left(H_{\alpha}\right)$ in $\mathfrak{m}_{\alpha}$ is a hypersurface of revolution about the line generated by $\sin \alpha \cdot S_{n}-\cos \alpha \cdot D$.

We set

$$
\begin{aligned}
e_{0} & =\sin \alpha \cdot S_{n}-\cos \alpha \cdot D, \\
e_{2 j-1} & =B_{j, n+1}, \\
e_{2 j} & =C_{j, n+1}, \quad j=1, \cdots, n .
\end{aligned}
$$

Then by Proposition 2, the complete conjugate locus is known by finding the conjugate points of $o$ along the geodesics emanating from $o$ with initial unit velocity vector:

$$
\xi_{\theta}=e_{0} \cos \theta+e_{1} \sin \theta, \quad \theta \in[-\pi, \pi] .
$$

$\xi_{\theta}$ is completed to an orthonormal basis of $\mathfrak{m}_{\alpha}$, viz., $\mathfrak{m}_{\alpha}=$ linear $\operatorname{span}\left\{\xi_{\theta}, \zeta_{\theta}=\right.$ $\left.-e_{0} \sin \theta+e_{1} \cos \theta, e_{2}, \cdots, e_{2 n}\right\}$.

Let $\varepsilon_{\theta}(t)$ denote the geodesic satisfying $\varepsilon_{\theta}(0)=o, \varepsilon_{\theta}^{\prime}(0)=\xi_{\theta}$. To solve Jacobi's equations along $\varepsilon_{0}(t)$, we write them in the canonical connection (of the second kind) - cf [3].

The torsion $T$ and curvature $B$, tensors of the canonical connection, are given at 0 by

$$
T(X, Y)=[X, Y]_{\mathfrak{m}_{\alpha}}, \quad B(X, Y) Z=\left[[X, Y]_{\mathfrak{h}_{\alpha}}, Z\right] .
$$

For future reference we list the torsion and curvature relative to the basis $e_{0}, \cdots, e_{2 n}$. First we set

$$
\beta=(n+1)(\sin \alpha) \alpha_{n}=\{2(n+1) / n\}^{1 / 2} \sin \alpha .
$$

Then

$$
\begin{aligned}
T\left(e_{0}, e_{2 k-1}\right) & =\beta e_{2 k}, \\
T\left(e_{0}, e_{2 k}\right) & =-\beta e_{2 k-1}, \\
T\left(e_{2 k-1}, e_{2 k}\right) & =\beta e_{0} .
\end{aligned}
$$

Otherwise, $T\left(e_{j}, e_{k}\right)=0, \quad B\left(e_{0}, e_{j}\right) \cdot e_{k}=B\left(e_{j}, e_{k}\right) \cdot e_{0}=0$ for all $j, k=$ $0,1, \cdots, 2 n$. Henceforth, assume $j \neq k$. Then

$$
B\left(e_{2 j-1}, e_{2 k-1}\right) \cdot\left\{\begin{array}{l}
e_{2 s-1}=-\delta_{k s} e_{2 j-1}+\delta_{j s} e_{2 k-1}, \\
e_{2 s}=\delta_{k s} e_{2 j}-\delta_{j s} e_{2 k},
\end{array}\right.
$$




$$
\begin{aligned}
& B\left(e_{2 j-1}, e_{2 j}\right) \cdot\left\{\begin{array}{l}
e_{2 s-1}=2 \delta_{j s} e_{2 j}+\left(2-\beta^{2}\right) e_{2 s}, \\
e_{2 s}=-2 \delta_{j s} e_{2 j-1}-\left(2-\beta^{2}\right) e_{2 s-1},
\end{array}\right. \\
& B\left(e_{2 j-1}, e_{2 k}\right) \cdot\left\{\begin{array}{l}
e_{2 s-1}=\delta_{k s} e_{2 j}+\delta_{j s} e_{2 k}, \\
e_{2 s}=-\delta_{k s} e_{2 j-1}-\delta_{j s} e_{2 k-1},
\end{array}\right. \\
& B\left(e_{2 j}, e_{2 k}\right) \cdot\left\{\begin{array}{l}
e_{2 s-1}=-\delta_{k s} e_{2 j-1}+\delta_{j s} e_{2 k-1}, \\
e_{2 s}=-\delta_{k s} e_{2 j}+\delta_{j s} e_{2 k} .
\end{array}\right.
\end{aligned}
$$

Now let $T_{\theta}$ of $B_{\theta}$ be the linear transformations given by

$$
\begin{aligned}
& T_{\theta} \cdot X=T\left(\xi_{\theta}, X\right), \\
& B_{\theta} \cdot X=B\left(\xi_{\theta}, X\right) \xi_{\theta} .
\end{aligned}
$$

Then relative to the basis $\zeta_{\theta}, e_{2}, \cdots, e_{2 n}$, we have

$$
\begin{aligned}
T_{\theta} \cdot \zeta_{0} & =\beta e_{2}, & B_{\theta} \cdot \zeta_{\theta} & =0, \\
T_{\theta} \cdot e_{2} & =-\beta \zeta_{0}, & B_{\theta} \cdot e_{2} & =\left(4-\beta^{2}\right)\left(\sin ^{2} \theta\right) e_{2}, \\
T_{\theta} \cdot e_{2 k-1} & =\beta(\cos \theta) e_{2 k}, & B_{\theta} \cdot e_{2 k-1} & =\left(\sin ^{2} \theta\right) e_{2 k-1}, \\
T_{0} \cdot e_{2 k} & =-\beta(\cos \theta) e_{2 k-1}, & B_{\theta} \cdot e_{2 k} & =\left(\sin ^{2} \theta\right) e_{2 k},
\end{aligned}
$$

where $k \geq 2$. In particular, $T_{\theta}$ and $B_{\theta}$ have the same invariant subspaces for all $\theta$.

As in [3], let $a_{i}(t), i=1, \cdots, 2 n$, be a parallel (with respect to the canonical connection) orthonormal frame along $\varepsilon_{\theta}(t)$ for which $a_{1}(0)=\zeta_{\theta}, a_{i}(0)=e_{i}$, $i=2, \cdots, 2 n$. If we write for any vector field $\eta(t)$ along $\varepsilon_{\theta}, \eta(t)=\sum_{i=1}^{2 n} \eta_{i}(t) a_{i}(t)$, then Jacobi's equations along $\varepsilon_{\theta}$ read as:

$$
\begin{gathered}
\left(\begin{array}{l}
\eta_{1} \\
\eta_{2}
\end{array}\right)^{\prime \prime}+\left(\begin{array}{cc}
0 & -\beta \\
\beta & 0
\end{array}\right)\left(\begin{array}{l}
\eta_{1} \\
\eta_{2}
\end{array}\right)+\left(\begin{array}{cc}
0 & 0 \\
0 & \left(4-\beta^{2}\right) \sin ^{2} \theta
\end{array}\right)\left(\begin{array}{l}
\eta_{1} \\
\eta_{2}
\end{array}\right)=0, \\
\left(\begin{array}{l}
\eta_{2 k-1} \\
\eta_{2 k}
\end{array}\right)^{\prime \prime}+\left(\begin{array}{cc}
0 & -\beta \cos \theta \\
\beta \cos \theta & 0
\end{array}\right)\left(\begin{array}{l}
\eta_{2 k-1} \\
\eta_{2 k}
\end{array}\right)+\left(\begin{array}{cc}
\sin ^{2} \theta & 0 \\
0 & \sin ^{2} \theta
\end{array}\right)\left(\begin{array}{l}
\eta_{2 k-1} \\
\eta_{2 k}
\end{array}\right)=0,
\end{gathered}
$$

$k=2, \cdots n$. Thus one obtains a basis of solutions vanishing at $t=0$, $\Lambda_{1}(t), \cdots, \Lambda_{2 n}(t)$ such that

$$
\left\langle\Lambda_{2 k-i}(t), a_{j}(t)\right\rangle=0,
$$

for all $t$, where $i=0,1 ; k=1, \cdots, n ; j=1, \cdots, 2 k-2,2 k+1, \cdots, 2 n$, and

$$
\left.\frac{D}{d t} \Lambda_{i}\right|_{t=0}=k_{i} a_{i}(0)
$$


$i=1, \cdots, 2 n, k_{i}=$ constant, where $D / d t$ denotes covariant differentiation in the canonical connection along $\varepsilon_{\theta}(t)$. Using the Remark of [4] and (20) one sees that $\Lambda_{2}, \cdots, \Lambda_{2 n}$ form a basis of isotropic Jacobi fields along $\varepsilon_{\theta}, \varepsilon_{\theta}^{\prime}(0) \neq$ $\pm e_{0}$; otherwise, no Jacobi fields are isotropic.

We turn to the solving of (17). Set

$$
\begin{aligned}
& \gamma=\left(4-\beta^{2}\right) \sin ^{2} \theta, \\
& \sigma=\left(4 \sin ^{2} \theta+\beta^{2} \cos ^{2} \theta\right)^{1 / 2} .
\end{aligned}
$$

Then the eigenvalues $\lambda$ of (17) are easily seen to be $\lambda^{2}=0$ and $\lambda= \pm \sqrt{-1} \sigma$. Standard calculation yields

$$
\begin{aligned}
& \Lambda_{1}(t)=\{-(\gamma / \beta) t-(\beta / \sigma) \sin \sigma t\} a_{1}(t)+\{1-\cos \sigma t\} a_{2}(t), \\
& \Lambda_{2}(t)=\{(\beta / \sigma)(1-\cos \sigma t)\} a_{1}(t)+(\sin \sigma t) a_{2}(t) .
\end{aligned}
$$

Conjugate points obtained from linear combinations of $\Lambda_{1}$ and $\Lambda_{2}$ have path values $t$ for which

$$
0=f(t)=(2 \beta / \sigma)(1-\cos \sigma t)+(\gamma / \beta) t \sin \sigma t .
$$

Also,

$$
f^{\prime}(t)=\left\{\left(2 \beta^{2}+\gamma\right) / \beta\right\} \sin \sigma t+(\gamma \sigma / \beta) t \sin \sigma t .
$$

Now $f(2 \pi k / \sigma)=0$ for all integers $k$. Indeed these values are precisely the zeros of $\Lambda_{2}(t)$. However, for sufficiently small $|t|>0, f(t)$ is given by $f(t)=$ $\{\beta \sigma+(\gamma \sigma / \beta)\} t^{2}+\cdots>0$; and $f^{\prime}(2 \pi k / \sigma)>0$ if and only if $\gamma(\theta) \neq 0$, i.e., $\xi_{\theta} \neq \pm e_{0}$. Thus, for $\xi_{\theta} \neq \pm e_{0}$ and every integer $k$, a linear combination of $\Lambda_{1}$ and $\Lambda_{2}$ vanishes for some $t_{0} \in(2 \pi k / \sigma, 2 \pi(k+1) / \sigma)$. Note that the Jacobi field in question is nonisotropic.

We now turn to (18). Since the matrices

$$
\mathscr{T}=\left(\begin{array}{cc}
0 & -\beta \cos \theta \\
\beta \cos \theta & 0
\end{array}\right), \quad \mathscr{B}=\left(\begin{array}{cc}
\sin ^{2} \theta & 0 \\
0 & \sin ^{2} \theta
\end{array}\right)
$$

commute, the system can be changed in the usual manner by letting

$$
\left(\begin{array}{l}
b_{2 k-1}(t) \\
b_{2 k}(t)
\end{array}\right)=\exp \left(\frac{t}{2} \mathscr{T}\right) \cdot\left(\begin{array}{l}
a_{2 k-1}(t) \\
a_{2 k}(t)
\end{array}\right)
$$

and $\eta=\hat{\eta}_{2 k-1} b_{2 k-1}+\hat{\eta}_{2 k} b_{2 k}$. The result is then

$$
\begin{aligned}
& \hat{\eta}_{2 k-1}^{\prime \prime}+\left(\sigma^{2} / 4\right) \hat{\eta}_{2 k-1}=0, \\
& \hat{\eta}_{2 k}^{\prime \prime}+\left(\sigma^{2} / 4\right) \hat{\eta}_{2 k}=0,
\end{aligned}
$$


and therefore $\Lambda_{2 k-1}(t)=\sin \sigma t / 2 \cdot b_{2 k-1}(t), \Lambda_{2 k}(t)=\sin \sigma t / 2 \cdot b_{2 k}(t)$. Geometrically, $b_{2 k-1}(t), b_{2 k}(t)$ are Riemannian-parallel orthonormal vector fields with initial values $e_{2 k-1}, e_{2 k}$ respectively, and $\sigma^{2} / 4$ is the sectional curvature of the 2-sections $\left(\varepsilon_{\theta}^{\prime}(t), b_{2 k-1}(t)\right)$ and $\left(\varepsilon_{\theta}^{\prime}(t), b_{2 k}(t)\right)$ for all $t$; cf. [3, p. 244]. The zeros of $\Lambda_{3}, \cdots, \Lambda_{2 n}$ have arc values $t=2 \pi k / \sigma$. We summarize our results in

Theorem 1. Let $\varepsilon:(-\infty, \infty) \rightarrow M_{\alpha}^{n} \cdot\left\|\varepsilon^{\prime}\right\|=1, \varepsilon(0)=o$ be a geodesic in $M_{\alpha}^{n}, \theta$ the angle from $e_{0}$ to $\varepsilon^{\prime}(0)$, and $\beta$ and $\sigma$ as given in (3) and (22) respectively. Then all isotropic Jacobi fields along $\varepsilon$ vanish for $t=2 \pi k / \sigma$, $k=0, \pm 1, \pm 2, \cdots$, and there exists a non-isotropic Jocobi field along $\varepsilon$ vanishing for $t=0$ and some $t_{0}<2 \pi / \sigma$ whenever $\varepsilon^{\prime}(0) \neq \pm e_{0}$. For $\varepsilon^{\prime}(0)=$ $\pm e_{0}$, no Jacobi fields along $\varepsilon$ are isotropic, and all Jacobi fields vanish for $t=2 \pi k / \sigma=2 \pi k / \beta$. The first conjugate locus of $o$ therefore consists entirely of non-isotropic conjugate points, and the generating set of the conjugate locus, in $\mathrm{m}_{\alpha}$, in the $\left(e_{0}, e_{1}\right)$-plane is given by the solutions of (25) for each $\theta$, where $\gamma(\theta), \sigma(\theta)$ are given by (21), (22). Finally, the generating set is symmetric with respect to the $e_{0}$-axis and $e_{1}$-axis.

\section{Totally geodesic submanifolds of constant curvature}

Let $\mathfrak{m}^{0}$ be a subspace of $\mathfrak{m}_{\alpha}$. Then Theorem 2 of [7] states that the subset $\operatorname{Exp} \mathfrak{m}^{0}$ (where Exp is the Riemannian exponential map) is a totally geodesic submanifold of $G / H$ if for every $X, Y, Z \in \mathfrak{m}^{0}, T(X, Y)$ and $B(X, Y) Z \in \mathfrak{m}^{0}$. (This is not $A$. Sagle's original statement of the theorem. To pass from his formulation to ours, one uses [3, (3)].) We remark that Exp $\mathrm{m}^{0}$ is homogeneous by (i) Sagle's explicit construction and (ii) an unpublished result of S. Kobayashi that every totally geodesic submanifold of a homogeneous space is homogeneous.

For each $k=1, \cdots, n$, let $V_{k}$ be the subspace of $\mathrm{m}_{\alpha}$ generated by $e_{2 k-1}, e_{2 k}$.

Theorem 2. Let $\mathfrak{m}^{0}$ be any subspace of $\mathfrak{m}_{\alpha}$ generated by unit vectors $X_{1}, \cdots, X_{k}$ where $X_{k} \in V_{k}, k=1, \cdots, n$. Then Exp $m^{0}$ is a totally geodesic submanifold, of constant curvature 1 , of $M_{\alpha}^{n}$. Furthermore, $n$ is the maximal dimension for the total geodesy of a submanifold of $M_{\alpha}^{n}$ of constant curvature 1 for all $\alpha$ when $n>1$, and for $\alpha<\pi / 2$ where $n=1$. Finally, the subspace $\mathrm{m}^{0}$ described is the only subspace of $\mathfrak{m}_{\alpha}$ generating a totally geodesic submanifold of constant curvature 1 through $o$.

Proof. First, for any $X, Y \in \mathfrak{m}^{0}, T(X, Y)=0$; and $X, Y, Z \in \mathfrak{m}^{0}$ implies $B(X, Y) Z \in \mathfrak{m}^{0}$ by checking (7)-(10). Also, since $T$ restricted to $\mathfrak{m}^{0} \times \mathfrak{m}^{0}$ vanishes identically, we have that $B(X, Y) Z=R(X, Y) Z$ for all $X, Y, Z \in \mathfrak{m}^{0}$, where $R(X, Y) Z$ denotes the Riemannian curvature tensor; cf. [3, (3)]. One checks that the Riemannian sectional curvature is 1 . To increase the dimension of $\mathfrak{m}^{0}$ would either (i) yield a non-trivial projection of $\mathfrak{m}^{0}$ onto $e_{0}$, which would contradict constant curvature assumption (if not also total geodesy), or (ii), for some $k=1, \cdots, n$, yield a projection of $\mathrm{m}^{0}$ onto all of $V_{k}$ which would 
contradict total geodesy by (6). The last statement in the theorem also follows from (i) and (ii).

\section{Riemannian curvature and the pinching of $M_{\alpha}^{n}$}

$R$ denotes the Riemannian curvature tensor of $M_{\alpha}^{n}, R_{\theta}$ the linear transformation $R_{\theta} \cdot Y=R\left(\xi_{\theta}, Y\right) \xi_{\theta}$. Then by (14) of [3], we have

$$
R_{\theta}=B_{\theta}-\frac{1}{4}\left(T_{\theta}\right)^{2},
$$

which implies

$$
\begin{aligned}
\boldsymbol{R}_{\theta} \cdot \zeta_{\theta} & =\left(\beta^{2} / 4\right) \cdot \zeta_{\theta}, \\
\boldsymbol{R}_{\theta} \cdot e_{2} & =\left\{\beta^{2} / 4+\left(4-\beta^{2}\right) \sin ^{2} \theta\right\} e_{2}, \\
\boldsymbol{R}_{\theta} \cdot e_{2 k-1} & =\left\{\beta^{2} / 4 \cos ^{2} \theta+\sin ^{2} \theta\right\} e_{2 k-1}, \\
\boldsymbol{R}_{\theta} \cdot e_{2 k} & =\left\{\beta^{2} / 4 \cos ^{2} \theta+\sin ^{2} \theta\right\} e_{2 k}, \quad k=2, \cdots, n .
\end{aligned}
$$

Therefore the set curv $\left(M_{\alpha}^{n}\right)$ of real numbers assumed as values of sectional curvatures is given by

$$
\operatorname{curv}\left(M_{\alpha}^{n}\right)=\left[(n+1) \sin ^{2} \alpha / 2 n, 4-\left(3(n+1) \sin ^{2} \alpha / 2 n\right)\right],
$$

and the pinching $\delta_{\alpha}^{n}$ is given by

$$
\delta_{\alpha}^{n}=\frac{(n+1) \sin ^{2} \alpha}{8 n-3(n+1) \sin ^{2} \alpha} .
$$

\section{Closed geodesics and Klingenberg's lemma}

We first note that by Theorem 2, all geodesics emanating from $o$ with initial velocity vector in the orthogonal complement of $e_{0}$ in $\mathfrak{m}_{\alpha}$ are simply closed and have length $2 \pi$.

We now note that $\exp \left(2 \pi \alpha_{n} / n\right) S_{n} \in S U(n)$, and that $2 \pi \alpha_{n} / n$ is the first value of $t$ for which $\exp t S_{n} \in S U(n)$. Also, recall that $\left[\mathfrak{h}_{\alpha}, S_{n}\right]=0$. Thus the group generated by $\mathfrak{h}_{\alpha} \oplus S_{n}=\mathfrak{a}_{n-1} \oplus \boldsymbol{R} \oplus \boldsymbol{R}$ is a cylinder with generator $S U(n) \times \boldsymbol{R}$ and base circle of length $2 \pi \alpha_{n} / n$. Now geodesics in $G_{n} / H_{\alpha}$ through $o$ are projections of the one parameter subgroups of $G_{n}$ generated by the elements of $\mathfrak{m}_{\alpha}$, and it is easy to see that $\gamma(t)=\pi\left(\exp t e_{0}\right)$ is a closed (and hence simply closed [6, Th. 3]) geodesic of length $\left(2 \pi \alpha_{n} / n\right) \sin \alpha$. Since the maximum curvature of $M_{\alpha}^{n}$ is given by $4-(3(n+1) / 2 n) \sin ^{2} \alpha$, Klingenberg's lemma [6, Th. 1] for odd dimensions would imply

$$
\left(2 \pi \alpha_{n} / n\right) \sin \alpha \geq 2 \pi /\left\{4-(3(n+1) / 2 n) \sin ^{2} \alpha\right\}^{1 / 2},
$$

from which one implies $\sin ^{2} \alpha \geq 2 n /(3 n+3)$. Thus for $\sin ^{2} \alpha<2 n /(3 n+3)$, Klingenberg's lemma is false. For $\sin ^{2} \alpha=2 n /(3 n+3)$ the pinching of $M_{\alpha}^{n}$ is $1 / 9$ for all $n$. One wonders... 


\section{Remarks}

(A) For any normal Riemannian homogeneous space $G / H$ with orthogonal decomposition $\mathfrak{g}=\mathfrak{h} \oplus \mathfrak{m}$, and for any $X \in m$, we let $T_{X}=T(X$,$) and$ $B_{X}=B(X)$,$X . We define G / H$ to be quasi-symmetric if for all $X \in m, T_{X}$ and $B_{X}$ commute. As noted in [3], $T_{X}$ is skew-symmetric and $B_{X}$ is symmetric. In a quasi-symmetric space, Jacobi's equations split into subsystems of 2 by 2's which are easily handled. Indeed, $M_{\alpha}^{n}$ is quasi-symmetric, and it is easy to see that for dimension 3 all $G / H$ are quasi-symmetric. Are all homogeneous $\boldsymbol{G} / \boldsymbol{H}$ quasi-symmetric?

(B) In [3] we proved that if every conjugate point of a simply connected normal Riemannian $G / H$ of rank one is isotorpic, then $G / H$ is homeomorphic to a symmetric space of rank one. For the spaces $M_{\alpha}^{n}$, one might say that the $A d\left(H_{\alpha}\right)$ acts almost transitively on $m_{\alpha}$. Yet, by Theorem 1, all the points of the first conjugate locus are non-isotropic. Also, if the linear isotropy is transitive on unit tangent spheres, the space is Riemannian symmetric (this is only known heretofore by classification arguments). We therefore

Conjecture. If every conjugate point of a simply connected normal Riemannian $\mathrm{G} / \mathrm{H}$ of rank one is isotropic, then $\mathrm{G} / \mathrm{H}$ is isometric to a Riemannian symmetric space of rank one

Added in proof. We note that A. Sagle's condition for total geodesy of submanifolds of a reductive Riemannian homogeneous space $G / H$ is only sufficient but not necessary. More preceisely, Sagle's theorem says what Kobayashi's does not, viz., if $T(X, Y), B(X, Y) Z \in \mathfrak{m}^{0}$ for all $X, Y, Z \in \mathfrak{m}^{0}$, then Exp $\mathrm{m}^{0}$ is homogeneous relative to a subgroup of $G$. Inspection as in the proof of Theorem 2 shows that we have indeed ennumerated all totally geodesic submanifolds of $G / H$ through $\pi(H)$.

\section{References}

[ 1 ] M. Berger, Les variétés riemanniennes homogènes normales simplement connexes a courbure strictement positive, Ann. Scuola Norm. Sup. Pisa 15 (1961) 179-246.

[2] — On the diameter of some Riemannian manifolds, Mimeographed notes, University of California, Berkeley, 1962.

[3] I. Chavel, Isotropic Jacobi fields, and Jacobi's equations on Riemannian homogeneous spaces, Comment. Math. Helv. 42 (1967) 101-112.

[ 4 ] - On normal Riemannian homogeneous spaces of rank one, Bull. Amer. Math. Soc. 73 (1967) 477-481.

[ 5 ] W. Klingenberg, Contributions to Riemannian geometry in the large, Ann. of Math. 69 (1959) 654-666.

[ 6 ] B. Kostant, On differential geometry and homogeneous spaces. I, II, Proc. Nat. Acad. Sci. U.S.A. 42 (1956) 258-261, 354-357.

[7] A. Sagle, $A$ note on triple systems and totally geodesic submanifolds in a homogeneous space, to appear in Nagoya Math. J. 